\title{
ECC
}

Eurasian Chemical Communications

\section{Nephrotic syndrome associated with carcinoma of unknown primary treated with chemotherapy: a rare case report}

\author{
Azita Zafar-Mohtashamia, Ali Bakhtiaria, Babak Hadian ${ }^{a, *}$, Zia Obeidavi ${ }^{b}$ \\ ${ }^{a}$ Assistant Professor, Department of Internal Medicine, Lorestan University of Medical Sciences, \\ Khorramabad, Iran
}

${ }^{b} M D$, Faculty of Medicine, Lorestan University of Medical Sciences, Khorramabad, Iran

Received: 18 August 2019, Accepted: 30 October 2019, Published: 02 November 2019

\begin{abstract}
Different glomerular diseases are associated with various neoplasms. It seems obvious that Carcinoma of Unknown Primary (CUP) induced nephrotic syndrome is rare. In this regard, the present article introduces a patient suffering from nephrotic syndrome associated with CUP. Patient is a 65-year-old non-smoker male who referred to the clinic for one-month generalized edema. The patient was also complaining from coughing and bulky yellowish sputum with no signs of blood. Patient's chest radiography revealed pulmonary pleural effusion of the left lung, but no other pathological findings were revealed. Patient's cytology report of pleural fluid indicated metastatic carcinoma, and therefore, the patient needed additional examinations so that the origin of metastases (lung, gastrointestinal tract, pancreas, etc.) could be detected. Finally, chemotherapy treatments began when the patient was diagnosed with Carcinoma of Unknown Primary (CUP). With the continuation of chemotherapy treatment and follow-up after more than a year of treatment, the patient is now generally well-off; his nephrotic syndrome has also been in remission parallel to chemotherapy. Significant point in the treatment of this patient is proteinuria, hypercholesterolemia and hyponatremia remission in two stages of chemotherapy. These findings suggest probable para-neoplastic nephrotic syndrome. The treatment of paraneoplastic is the treatment of underlying cause.
\end{abstract}

Keywords: Nephrotic syndrome; paraneoplastic syndromes; neoplasms.

\section{Introduction}

Paraneoplastic syndromes will result in the emergence of clinical manifestations that are not directly related to tumors, local invasion or metastases, and they are developed due to secretions from tumor cells such as hormones, cytokines, growth factors, and tumor antigens [1]. After the paraneoplastic syndromes were presented and parallel to it in 1922 a researcher named Galloway coined the new phrase of "paraneoplastic glomerulopathy" in medical science by introducing a new nephrotic syndrome associated with Hodgkin's disease [2]. After Galloway, many writers began to introduce patients who were simultaneously suffering from both 
cancer and glomerular disease, but the causal relationship between the two was still unknown [3].

Diagnosis of paraneoplastic syndrome is based on quadruple criteria, which include: a) no other specific cause for the syndrome concerned; b) a time relation between the diagnosis of the syndrome and the cancer; c) clinical and histological remission after complete tumor removal by surgery; or complete remission following chemotherapy; d) tumor recurrence in conjunction with rise in associated symptoms $[1,4]$.

Glomerulopathy is a general term for describing glomerular damages. Nephrotic syndrome is one of the most common clinical manifestations of glomerulopathy which is identified through severe proteinuria (more than $3.5 \mathrm{~g}$ of protein in 24-hour urine), hypoalbuminemia (less than $30 \mathrm{~g} / \mathrm{l}$ ), high cholesterol and edema [5]. Various predisposing factors such as primary and systemic kidney disease, as well as secondary causes such as congenital infections, diabetes, systemic lupus erythematosus (SLE), neoplasms or using a specific drug can lead to the occurrence of nephrotic syndrome with histopathological variable patterns [3,5-7].

Different glomerular diseases are associated with various neoplasms. Nephrotic syndrome caused by membranous nephropathy is associated with solid tumors, cases of Minimal Change Disease (MCD), IgA Nephropathy, Focal Segmental Glomerulosclerosis (FSGS), Membranoproliferative Glomerulonephr itis (MPGN), Rapidly Progressive Glomerulonephritis

(RPGN), Amyloidosis, and Thrombotic Microangiopathy (TMA) associated with Solid tumors have also been reported. The most common neoplasms associated with paraneoplastic glomerular diseases are lung and gastrointestinal carcinoma [8]. MCD is strongly associated with Hodgkin's lymphoma [9]. However, it seems obvious that Carcinoma of Unknown Primary (CUP) induced nephrotic syndrome is rare; in this regard, the present article introduces a patient suffering from nephrotic syndrome associated with CUP, which improved after the cancer treatment.

\section{Case report}

Patient is a 65-year-old non-smoker male who referred to the clinic of Shahid Rahimi Hospital of Khorramabad which is associated to Lorestan University of Medical Sciences for onemonth generalized edema. The patient have been diagnosed with type 2 diabetes (under treatment with injecting insulin) and hypertension since 10 years ago, and he had undergone Coronary Artery Bypass Graft (CABG) surgery three months before referral and he had no complications after surgery. Before CABG examinations revealed no evidence of nephrotic syndrome. The patient also had a history of Hypersensitivity Pneumonitis and consumption of Atrovent and Seroflo sprays and was treated with prazosin and tamsulosin due to $\mathrm{BPH}$. He also noted the history of kidney stones. His family history was negative. The patient was also complaining from coughing and bulky yellowish sputum with no signs of blood. The admitted patient vital sign was a blood pressure of $130 / 80 \mathrm{mmHg}$, a heart rate of 86 beats per minute, a breathing rate of 14 per minute, and a body temperature of 37.2 degrees Celsius. His height was $171.2 \mathrm{~cm}$ and his weight was $86.24 \mathrm{~kg}$. The patient had a weight gain of 10 kilograms over the past month. The patient was fully awake and no abnormalities were observed in his mental status assessment. 
The patient did not have icteric sclera and pale conjunctiva. His lymph nodes were not touchable. Through the auscultation of the respiratory system through the lung fields, a decrease in the pulmonary sounds was heard in the lower left lung. Cutaneous (pitting) edema was observed in both lower limbs of the patient.

The results of the laboratory tests included white blood cell count (5.2 $\left.\times 1000 / \mathrm{mm}^{3}\right)$, hemoglobin count $(13.7$ $\mathrm{g} / \mathrm{dL})$ and platelet count (145 $\times 1000 / \mathrm{mm}^{3}$ ) in the form of Complete Blood Count (CBC) and urea (62 $\mathrm{mg} / \mathrm{dL})$, creatinine $(1.2 \mathrm{mg} / \mathrm{dL})$, total protein $(2.86 \mathrm{~g} / \mathrm{dL})$, albumin $(2 \mathrm{~g} / \mathrm{dL})$, total bilirubin $(1.02 \mathrm{mg} / \mathrm{dL})$, aspartate transaminase (14 U/L), alanine transaminase (5 U/L), alkaline phosphatase (208 U/L), total cholesterol (189 mg/dL), sodium (129 mEq/L), and potassium $(4.4 \mathrm{mEq} / \mathrm{L})$. Albuminuria $4+$ and hematuria $4+$ were also visible in the urinalysis. The 24-hour urine collection also showed proteinuria in the range of nephrotic syndrome $(4.5 \mathrm{~g} / 24 \mathrm{~h})$. Serological markers of hepatitis B and C were negative. Rheumatoid factor and Anti-dsDNA antibody were also negative. Monoclonality (M-spike) was not revealed in serum and urine electrophoresis. Other laboratory examinations of the patient (including PSA levels) were normal. Patient's chest radiography revealed pulmonary pleural effusion of the left lung, but no other pathological findings were revealed. Patient's cytology report of pleural fluid (pleural fluid of the patient was transudative) indicated metastatic carcinoma (Signet Ring Cell Carcinoma (SRCC), that is a rare form of highly malignant adenocarcinoma), and therefore; the patient needed additional examinations so that the origin of metastases (lung, gastrointestinal tract, pancreas, etc.) could be detected.
Chest CT Scan was done, which revealed evidences of hyperinflation and emphysema in the field of both lungs (small airway disease). Moderate pleural effusion was observed in the left lung with collapse in the base of the left lung and a $13 \times 19 \mathrm{~mm}$ nodular fibrosis was observed in the upper lobe of the left lung (Figure 1).

The patient was then subjected to a lung needle biopsy (the obtained biopsy specimen included lung parenchyma along with fibrosis and anthracosis) and no infiltration of the tumor or granulomatous tissue was visible. Moreover, the patient received abdominal and pelvic CT scans which revealed no other abnormal findings except for a few cortical cysts with a maximum diameter of $40 \mathrm{~mm}$ in the left kidney and $30 \mathrm{~mm}$ in the right kidney (Figure 2).

In pelvic MRI without contrasts, any other findings which indicate malignancy were not reported except for the enlargement of the prostate that could have been due to BPH. In order to make sure that the prostate enlargement was not malignant, the patient was subjected to prostate biopsy and the results indicated nodular hyperplasia. Gastrointestinal endoscopy was performed, and the findings indicated erosive gastritis and nothing but hemorrhoid was reported in the colonoscopy examination. Finally, the patient was diagnosed with CUP. Chemotherapy treatments was begun with Taxotere, Cisplatin, and Fluorouracil.

After two courses of chemotherapy the nephrotic range of proteinuria, hypercholesterolemia, and hyponathremia were resolved. Followup after more than a year of treatment, reveal that the patient is good and in remission. These findings suggest that nephrotic syndrome is paraneoplastic. 

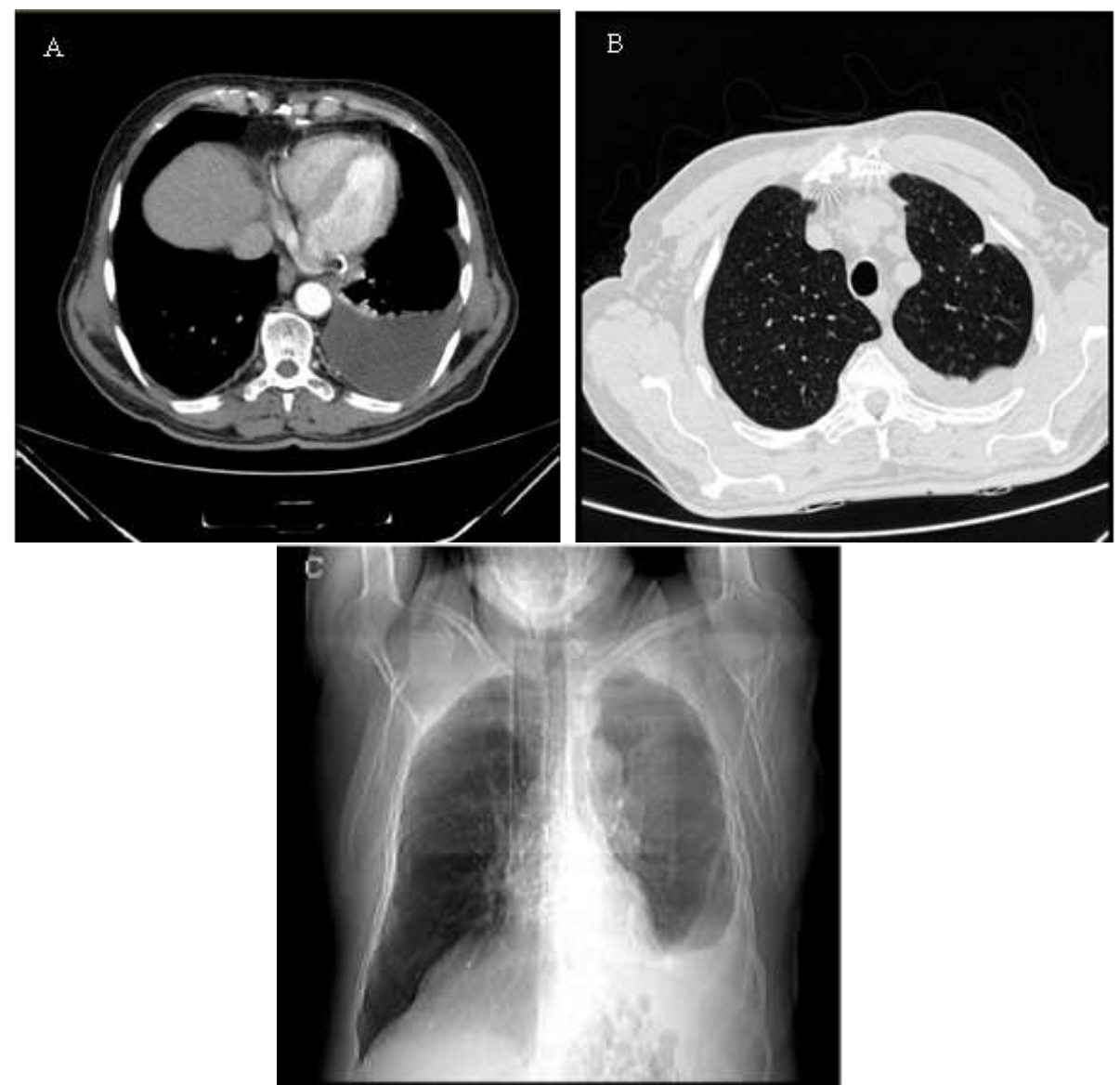

Figure 1. Chest Computed Tomography scan of the patient. (A) Transverse plane view (Mediastinal window). (B) Transverse plane view (Lung Window). (C) Coronal plane view
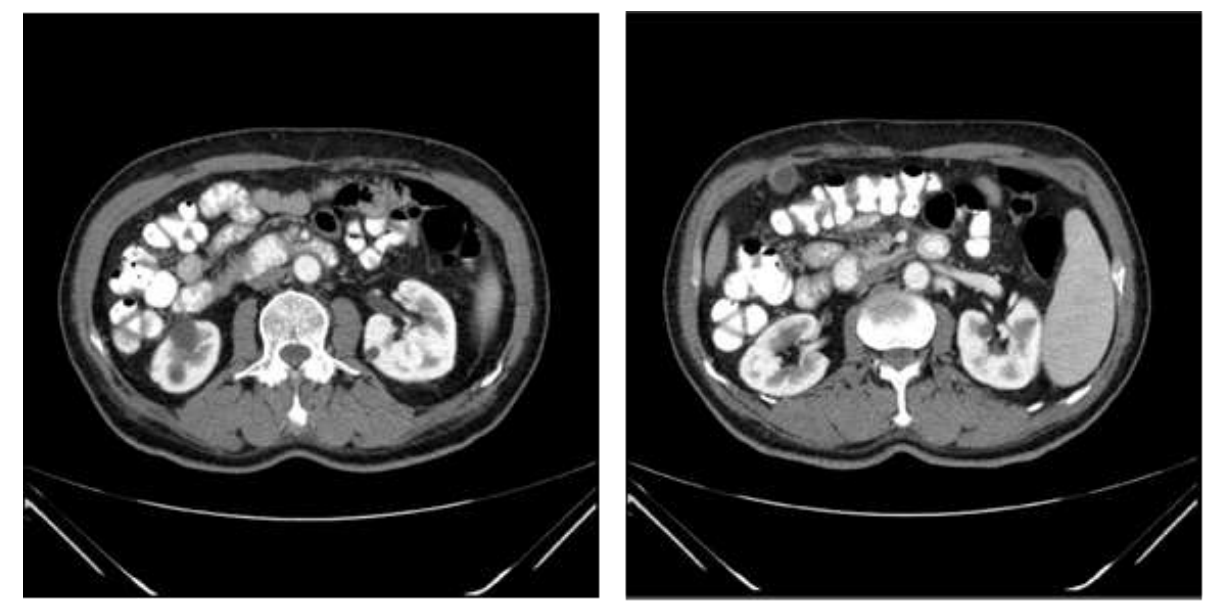

Figure 2. Abdominal Computed Tomography scan of the patient (Transverse plane view)

\section{Discussion and conclusion}

Paraneoplastic glomerulonephritis was first raised about 40 years ago by Lee et al. [10]. Subsequently, membrane nephropathy associated with Solid tumors and Minimal Change Disease associated with Hodgkin's lymphoma were recognized as classical paraneoplastic glomerulonephritis. Other glomerulonephritis such as FSGS, Membranoproliferative

Glomerulonephritis, IgA Nephropathy, and Rapidly Progressive Glomerulonephritis (RPGN) are also 
associated with malignancies. The treatment of paraneoplastic glomerulonephritis is completely different from the treatment of idiopathic glomerulonephritis, which is why diagnosis of paraneoplastic glomerulonephritis is of great importance [3,11].

Nephrotic syndrome includes a set of symptoms caused by damage to the basement membrane of the kidney glomeruli. Some of the causes of nephrotic syndrome are diabetes, systemic lupus erythematosus, sarcoidosis, amyloidosis, cancer, hepatitis $B$ and $C$ [3,5-7]. In the present patient, the cause of the disease was Metastatic Carcinoma of Unknown Primary.

Carcinoma of Unknown Primary consists of a diverse group of cancers and is defined as a metastatic disease without a specified primary tumor at the time of initial emergence [12]. According to available statistics, CUP accounts for about $2-5 \%$ of all cancers. The advancement made in the diagnostic and therapeutic methods has failed to significantly affect the challenging diagnosis of CUP. CUP diagnostic criteria such as biopsy confirms the malignancy for which a specific primary origin was not found after filling medical history forms completely, performing complete physical examinations, evaluating the function of kidneys and liver, performing blood tests, chest X-ray test, abdominal and pelvic CT scans, as well as mammogram or Prostate Specific Antigen (PSA) test [13]. In the introduced patient, the primary origin of metastatic carcinoma of the pleura was not found despite filling the medical history completely, performing full physical examinations, as well as laboratory examinations and full imaging.
Chemotherapy is the main treatment for most patients with CUP, performing surgery, radiotherapy, and periodic monitoring of patients may also be helpful $[14,15]$. The treatment of known cancer, often lead to improvement of renal disease.

Treatment for paraneoplastic nephrotic syndrome is complicated. Some studies have indicated the effectiveness of surgical treatment in curing paraneoplastic nephrotic syndrome $[16,17]$. However, there is no standard treatment for paraneoplastic nephrotic syndrome in nonsurgically malignant cases. Shikata et al. (2000) introduced two patients with nephrotic syndrome associated with advanced localized lung cancer and reported that the trace of urine protein and edema in these patients were significantly in remission after radiotherapy [3]. Furthermore, Lin et al. introduced a patient with non-small cell lung cancer (NSCLC) and noted that nephrotic syndrome in this patient was significantly in remission after radiotherapy of lung tumor in pulmonary [18]. Miyajima et al. have also introduced a patient with advanced localized lung adenocarcinoma who responded to chemoradiotherapy [19]. Mentioned studies would suggest in some way that radiotherapy and chemoradiotherapy can be used as a selective treatment for some types of advanced localized lung cancers in a patient with paraneoplastic nephrotic syndrome; as in the introduced patient, chemotherapy treatment of metastatic adenocarcinoma of pleura resulted in the remission based on clinical symptoms and laboratory findings associated with nephrotic syndrome.

\section{Conclusion}

Nephrotic syndrome can be the paraneoplastic symptom of CUP. So even in diabetic patients with nephrotic 
range proteinuria that is inappropriate with diabetic course, other causes of nephrotic syndrome, including CUP must be considered.

\section{Conflict of interests}

The authors declare no conflict of interests.

\section{References}

[1] P.M. Ronco, Kidney Int., 1999, 56, 355-377.

[2] J. Galloway, Br. Med. J., 1922, 23, 1201-1204.

[3] J. Bacchetta, L. Juillard, P. Cochat, J.P. Droz, Critical Reviews in Oncology/Hematology, 2009, 70, 39-58.

[4] F. Tourneur, R. Bouvier, J, Langue, M.H. Saïd, C. Bergeron, M. Hermier, P. Cochat, Pediatr. Nephrol., 2000, 14, 5355.

[5] (a) S.R. Orth, E. Ritz, N. Engl. J. Med., 1998, 338, 1202-1211. (b) R. Jamili Oskouei, N. Moradi-Kor, S. Abbasi Maleki, Am. J. Cancer. Res., 2017, 7: 610-627.

[6] K.D. Jhaveri, H.H. Shah, K. Calderon, E.S. Campenot, J. Radhakrishnan, Kidney Int., 2013, 84, 34-44.

[7] T. Soleymanian, G. Hamid, M. Arefi, I. Najafi, M.R. Ganji, M. Amini, M. Hakemi, M.R. Tehrani, B. Larijani, Renal failure, 2015, 37, 572-575.

[8] A.M. Davison, Nephrol. Dial. Transplant, 2001, 16, 13-14.

[9] V. Audard, F. Larousserie, P. Grimbert, M. Abtahi, J.J. Sotto, A. Delmer, F. Boue, D. Nochy, N. Brousse, R. Delaru, P. Remy, P. Ronco, D. Sahali, P. Lang, O. Hermine, Kidney Int., 2006, $69,2251-2260$.
[10] J.C. Lee, H. Yamauchi, J.J. Hopper, Ann. Intern. Med., 1966, 64, 4151.

[11] (a) Y.H. Lien, L.W. Lai, Nat. Rev. Nephrol., 2011, 7, 85-95. (b) P. Saraei, I. Asadi, M.A. Kakar, N. Moradi-Kor, Cancer. Manag. Res., 2019, 11, 32953313.

[12] J.L. Abbruzzese, R. Lenzi, M.N. Raber, S. Pathac, P. Frost, Semin. Oncol., 1993, 20, 238-243.

[13] R. Bugat, A. Bataillard, T. Lesimple, J.J. Voigt, S. Culine, A. Lortholary, Y. Merrouche, G. Ganem, M.C. Kaminsky, S. Negrier, M. Perol, C. Laforet, P. Bedossa, G. Bertrand, J.M. Coindre, K. Fizazi, Br. J. Cancer, 2003, 89, S59-S66.

[14] G.R. Varadhachary, Gastrointestinal Cancer Research, 2007, 1, 229-235.

[15] K. Ghanadi, H. Mahmoudvand, Al. Bakhtiari, M. Gorji, N. Moradi-kor, M.J. Tarahi, M. Garmsiri, Z. Obeidavi, Biomol Concepts, 2019, 10, 68-72.

[16] W.H. Coltharp, S.M. Lee, R.F. Miller, M.S. Averbuch, Ann. Thorac. Surg., 1991, 51, 308-309.

[17] (a) S.G. Pauker, R.I. Kopelman, N. Engl. J. Med., 1993, 328, 1621-1624. (b) M. Karimi Goftar, N. Moradi-Kor, Z. Moradi Kor, Int. J. Adv. Biol. Biom. Res., 2014, 2, 811-822.

[18] F.C. Lin, J.Y. Chen, A.H. Yang, S.C. Chang, Am. J. Med. Sci., 2002, 324, 161-165.

[19] S. Miyajima, Y. Taguchi, E. Tanaka, T. Inoue, M. Sakuramoto, M. Minakuchi, Y. Maeda, K. Maniwa, K. Tanizawa, M. Okamoto, T. Takeda, Nihon Kokyuki Gakkai Zasshi, 2006, 44, 631-635.

How to cite this manuscript: Azita Zafar-Mohtashami, Ali Bakhtiari, Babak Hadian, Zia Obeidavi. "Nephrotic syndrome associated with carcinoma of unknown primary treated with chemotherapy: a rare case report". Eurasian Chemical Communications, 2020, 2(2), 196-201. 